\title{
Improved Methods to Produce Tissue-Engineered Skin Substitutes Suitable for the Permanent Closure of Full-Thickness Skin Injuries
}

Danielle Larouche, ${ }^{1,2}$ Laurence Cantin-Warren, ${ }^{1,2}$ Maxime Desgagné, ${ }^{1,2}$ Rina Guignard ${ }^{1,2}$ Israël Martel, ${ }^{1,2}$ Akram Ayoub, Amélie Lavoie, ${ }^{1,2}$ Robert Gauvin, ${ }^{1-3}$ François A. Auger, ${ }^{1,2 \dagger}$ Véronique J. Moulin, ${ }^{1,2, \dagger}$ and Lucie Germain ${ }^{1,2, \dagger, *}$

\begin{abstract}
There is a clinical need for skin substitutes to replace full-thickness skin loss. Our group has developed a bilayered skin substitute produced from the patient's own fibroblasts and keratinocytes referred to as Self-Assembled Skin Substitute (SASS). After cell isolation and expansion, the current time required to produce SASS is 45 days. We aimed to optimize the manufacturing process to standardize the production of SASS and to reduce production time. The new approach consisted in seeding keratinocytes on a fibroblast-derived tissue sheet before its detachment from the culture plate. Four days following keratinocyte seeding, the resulting tissue was stacked on two fibroblast-derived tissue sheets and cultured at the air-liquid interface for 10 days. The resulting total production time was 31 days. An alternative method adapted to more contractile fibroblasts was also developed. It consisted in adding a peripheral frame before seeding fibroblasts in the culture plate. SASSs produced by both new methods shared similar histology, contractile behavior in vitro and in vivo evolution after grafting onto mice when compared with SASSs produced by the 45-day standard method. In conclusion, the new approach for the production of high-quality human skin substitutes should allow an earlier autologous grafting for the treatment of severely burned patients.
\end{abstract}

Keywords: autologous; regenerative medicine; skin equivalent; tissue culture; tissue therapy; transplants

\section{Introduction}

The standard surgical treatment for the permanent closure of large full-thickness skin wounds that can occur following acute trauma or surgical intervention consists in replacing tissue loss with skin autografts harvested from an uninjured donor site on the patient. However, when the skin loss exceeds $50 \%$ of the total body surface area of the patient, donor site availability for harvesting autografts becomes limited. Progress in tissue engineering has led to the development of technologies, allowing the production of skin substitutes. These substitutes can be either epidermal or dermal substitutes or bilayered skin substitutes.
Tissue-engineered bilayered skin substitutes are composed of both a dermis and an epidermis. The ideal bilayered skin substitute should be easy to handle, must permanently ensure the skin barrier function, and should not induce a host immune rejection. Some models of bilayered skin substitutes produced in the laboratory have been reported to allow permanent coverage of full-thickness wound, to reduce the need for harvesting autografts, and are indicated as an adjunct treatment for massive burns. ${ }^{1-3}$

The self-assembly approach developed at the LOEX $^{4,5}$ allows for the reconstruction of a fully autologous bilayered skin substitute. This method is based

\footnotetext{
'Département de Chirurgie, Faculté de Médecine, Centre de Recherche en Organogénèse Expérimentale de I'Université Laval/LOEX, Université Laval, Québec, Canada. ${ }^{2}$ Centre de Recherche du CHU de Québec-Université Laval, Axe Médecine Régénératrice, Québec, Canada.

${ }^{3}$ Centre Québécois sur les Matériaux Fonctionnels (CQMF), Québec, Canada.

†Co-senior authors.

*Address correspondence to: Lucie Germain, PhD, Centre de Recherche du CHU de Québec-Université Laval, Axe Médecine Régénératrice, LOEX, Aile-R, 1401, 18e rue, Québec G1J 1Z4, Canada, E-mail: lucie.germain@fmed.ulaval.ca
}

(C) Danielle Larouche et al. 2016; Published by Mary Ann Liebert, Inc. This Open Access article is distributed under the terms of the Creative Commons License (http://creativecommons.org/licenses/by/4.0), which permits unrestricted use, distribution, and reproduction in any medium, provided the original work is properly credited. 
on the capability of cells to form an organized threedimensional tissue without using any exogenous scaffold or biomaterial. The resulting skin tissue shares many properties with native human skin and minimizes the host response after transplantation. The self-assembly method generates a highly functional and mechanically stable skin substitute ${ }^{6}$ preserving epithelial stem cells, ${ }^{7}$ which is suitable for autologous grafting in humans. ${ }^{8}$

The first published self-assembly method involves a 45-day production period that includes the fabrication of the dermal component (28 days), the seeding of keratinocytes followed by a 7-day submerged culture period, and maturation at the air-liquid interface for an additional 10 days. ${ }^{9,10}$

The aim of the present study was to reduce the production time of Self-Assembled Skin Substitute (SASS) and to standardize the manufacturing protocol for clinical production purposes. The new method only requires a 31-day production period. The resulting SASSs are equivalent in terms of histological properties, contractility, and in vivo evolution compared with SASSs produced with the 45-day reference method.

\section{Materials and Methods}

\section{Cell populations}

The study was approved by the institutional animal care and use committee and by the institutional committee for the protection of human subjects. The procedures followed were in accordance with the Helsinki Declaration of 1975.

\section{Cell isolation and culture}

Human keratinocytes and dermal fibroblasts were isolated from 1 newborn and 11 adult (18 to 46 years old) human skin samples as previously described. ${ }^{10}$ Dermal fibroblasts were cultured in fibroblast medium (Dulbecco-Vogt modified Eagle medium [Corning] supplemented with $10 \%$ fetal bovine serum [FBS; Seradigm], $100 \mathrm{U} / \mathrm{mL}$ penicillin [Pharmaceutical Partners of Canada, Inc.], and $25 \mu \mathrm{g} / \mathrm{mL}$ gentamycin [Galenova]). Keratinocytes were grown on a feeder layer of irradiated human fibroblasts ${ }^{11}$ and cultured in keratinocyte medium (Dulbecco-Vogt modified Eagle medium: Ham's F12, ratio 3:1, $24.3 \mu \mathrm{g} / \mathrm{mL}$ adenine [Corning] supplemented with $5 \mu \mathrm{g} / \mathrm{mL}$ insulin [Sigma-Aldrich], $1.1 \mathrm{mM}$ hydrocortisone [Novapharm], $0.212 \mu \mathrm{g} / \mathrm{mL}$ isoproterenol hydrochloride [Sandoz], 5\% bovine Fetal Clone II serum [HyClone], $10 \mathrm{ng} / \mathrm{mL}$ human epidermal growth factor [R\&D Systems], $100 \mathrm{U} / \mathrm{mL}$ penicillin, and $25 \mu \mathrm{g} / \mathrm{mL}$ gentamycin). For tissue production, cells were used at passage three for keratinocytes and passage two to six for fibroblasts.

\section{Skin substitute production}

The tissue-engineered skin methods presented here derive from the SASS method described earlier, ${ }^{10}$ here after referred to as SASS-1. In this study, tissue-engineered skin substitutes produced by three adaptations of the SASS-1 method were compared: the method referred to as SASS-2 (reference method, previously presented in Ref. $\left.{ }^{6}\right)$, SASS-3, and SASS-4. Each proposed method was performed 6-10 times in triplicate using different combinations of fibroblasts and keratinocytes, of which four combinations were donor matched.

Fibroblasts were seeded at a density of $4 \times 10^{3}$ cells/ $\mathrm{cm}^{2}$ in $85 \mathrm{~cm}^{2} \mathrm{Nunc}^{\mathrm{TM}}$ Omnitray ${ }^{\mathrm{TM}}$ tissue culture plate with a removable lid (Fisher Scientific) and cultured in fibroblast medium containing $50 \mu \mathrm{g} / \mathrm{mL}$ ascorbic acid (Galenova) to promote extracellular matrix production.

The method SASS-2 consists of placing a custommade frame (inner dimensions: $46 \times 76 \mathrm{~mm}$, outer dimensions: $62 \times 99 \mathrm{~mm}$ ) cut out of Ahlstrom grade 237 filter paper (Fisher Scientific) onto a 21-day fibroblastderived tissue sheet still attached to the bottom of the culture plate. Tissue surrounding the frame is folded thereon. The frame is grasped with forceps, and the tissue is carefully detached and stacked on a subsequent tissue sheet. Surrounding tissue is again folded onto the frame, and the second tissue sheet is detached. The procedure is repeated with a third fibroblast-derived tissue sheet. Then, the stack of three sheets is attached to the paper frame using LIGACLIPS ${ }^{\circledR}$ (Ethicon EndoSurgery) and placed in a $150 \mathrm{~mm}$ diameter culture Petri dish (Corning). For the first $24 \mathrm{~h}$, a surgical sponge (Merocel $^{\circledR}$; Medtronic, Instruments Ophtalmiques INNOVA) is installed on the top of stacked sheets and held in place by stainless steel weights. After 7 days of culture in fibroblast medium containing $50 \mu \mathrm{g} / \mathrm{mL}$ ascorbic acid, 0.9 to $2 \times 10^{5}$ keratinocytes $/ \mathrm{cm}^{2}$ are seeded within a stainless steel seeding mold (inner dimensions: $48 \times 76 \mathrm{~mm}$, outer dimensions: $50 \times 78 \mathrm{~mm}$ ) placed on the top of the stacked tissue sheets. After 7 days of culture submerged in keratinocyte medium containing $50 \mu \mathrm{g} / \mathrm{mL}$ ascorbic acid, the resulting tissue is detached from the bottom of the culture plate and raised on a custommade frame (inner dimensions: $46 \times 76 \mathrm{~mm}$, outer dimensions: $96 \times 113 \mathrm{~mm}$ ) cut in filter paper (Ahlstrom 237 ) or onto a $100 \times 100 \mathrm{~mm}$ polypropylene membrane (Spectra/Mesh ${ }^{\circledR}$ Woven polypropylene membrane filters, mesh opening: $500 \mu \mathrm{m}$, open area: $2 \%$, thickness: 
$610 \mu \mathrm{m}$, Spectrumlabs.com). The construct is then transferred on a custom-made acrylic support to maintain the tissue at the air-liquid interface in a $150 \mathrm{~mm}$ diameter culture Petri dish. The tissue is further cultured for 10 days at the air-liquid interface in keratinocyte medium exempt of epidermal growth factor and containing $50 \mu \mathrm{g} / \mathrm{mL}$ ascorbic acid. The resulting skin substitute is referred to as SASS-2. The total production time is 45 days (Fig. 1A).

The method SASS- 3 consists in seeding 0.9 to $2 \times 10^{5}$ keratinocytes $/ \mathrm{cm}^{2}$ directly onto the top of a 17-day fibroblast-derived tissue sheet, produced as described above, still attached to the bottom of the culture plate. The tissue is then cultured in keratinocyte medium containing $50 \mu \mathrm{g} / \mathrm{mL}$ ascorbic acid, and medium was changed once a day. After 4 days, a custom-made filter paper frame (peripheral anchorage inner dimensions: $46 \times 76 \mathrm{~mm}$, outer dimensions: $62 \times 99 \mathrm{~mm}$, Ahlstrom grade 237 filter paper) is placed onto the tissue sheet containing keratinocytes and fibroblasts. The tis- sue adheres to the frame. The thin strip $(2-4 \mathrm{~mm})$ of tissue surrounding the frame is folded thereon without covering the inside tissue. Both extremities of the frame are grasped with forceps, and the tissue is progressively raised. Once detached, the tissue is stacked onto a 21 day fibroblast-derived tissue sheet. Surrounding tissue is folded onto the frame without covering the inside tissue, and the second tissue sheet is detached. The procedure is repeated with another 21-day fibroblast-derived tissue sheet, and the three-sheet stacked tissue is fixed to the frame using LIGACLIPS (Ethicon Endo-Surgery). The construct is transferred onto a polypropylene membrane laid on a custom-made acrylic support to hold the tissue at the air-liquid interface in a $150 \mathrm{~mm}$ diameter culture Petri dish and further cultured 10 days at the air-liquid interface as described above. The resulting skin substitute is referred to as SASS-3. The total production time is 31 days (Fig. 1B).

According to the patient's cells, dermal fibroblasts can sometimes induce contraction of unanchored tissue

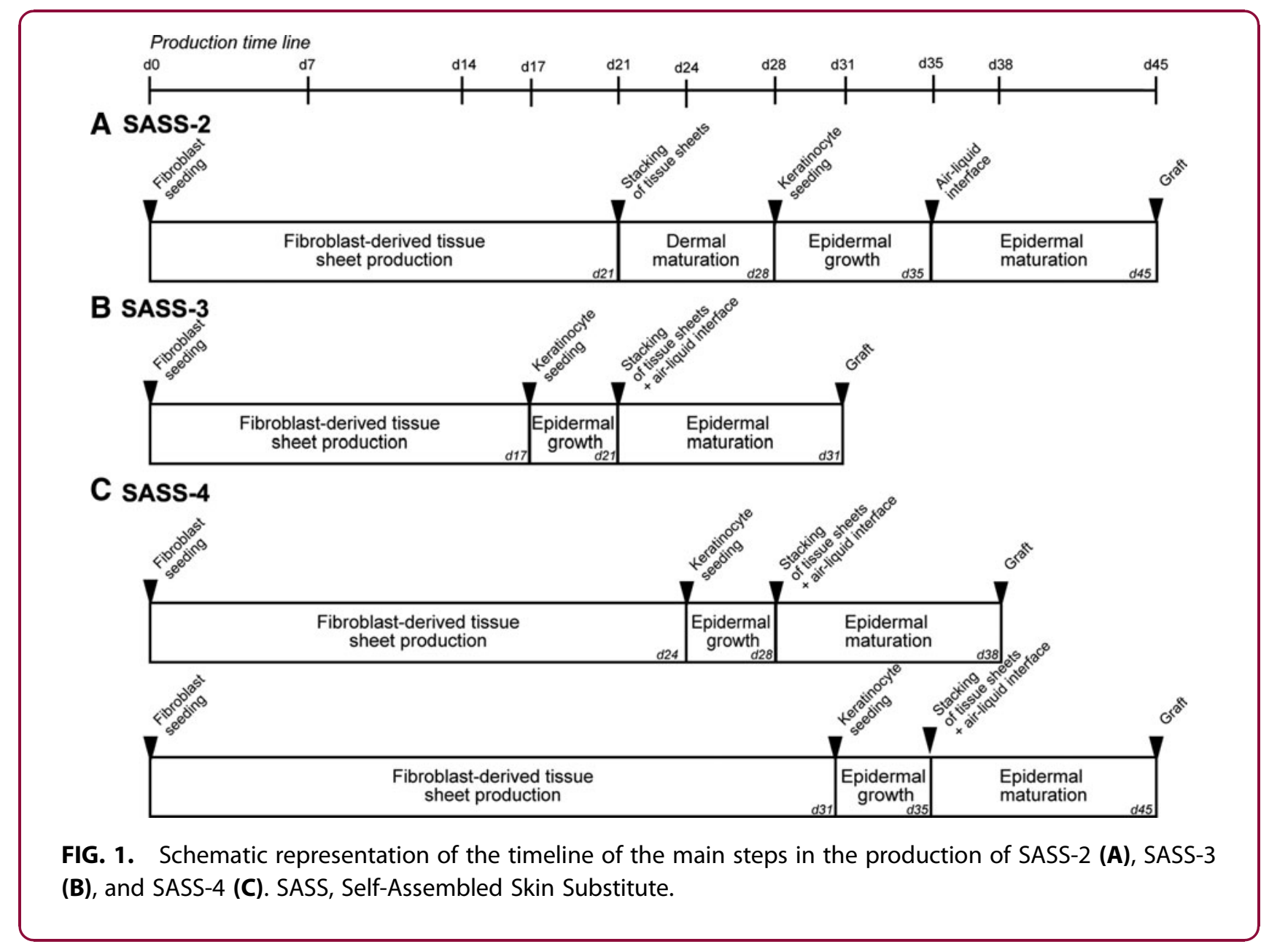


sheets when keratinocytes were added on the sheet. To circumvent this problem, an alternative approach referred to as method SASS-4 was developed. It consists of placing a custom-made frame (inner dimensions: $57 \times 84 \mathrm{~mm}$, outer dimensions: $73 \times 102 \mathrm{~mm}$ ) cut out of filter paper (Ahlstrom grade 610 filter paper [Fisher Scientific]) into the culture plate before seeding the fibroblasts. $^{12,13}$ Thus, cells grow in the culture plate and adhere to the peripheral paper anchorage. Cells are cultured in fibroblast medium containing $50 \mu \mathrm{g} /$ $\mathrm{mL}$ ascorbic acid for typically $24-31$ days. Then, 0.9 to $2 \times 10^{5}$ keratinocytes $/ \mathrm{cm}^{2}$ are seeded directly onto the top of an anchored fibroblast-derived tissue sheet still attached to the bottom of the culture plate. The tissue is then cultured in keratinocyte medium containing $50 \mu \mathrm{g} / \mathrm{mL}$ ascorbic acid, and the medium was changed once a day. After 4 days, the tissue is carefully detached and stacked with two other anchored fibroblast tissue sheets. The remaining steps are identical to those of the method SASS-3. The resulting skin substitute is referred to as SASS-4. The total production time is $38-45$ days (Fig. 1C).

All cultures and skin substitutes were kept at $37^{\circ} \mathrm{C}$ in a humidified incubator containing $8 \% \mathrm{CO}_{2}$, and the culture medium was changed three times per week except when otherwise indicated.

\section{Contraction kinetic on agar-gelled substrate}

The structural stability of the skin substitutes was assessed at the end of the culture period (after 10 days of culture at the air-liquid interface) by measuring tissue contraction with the method of agar-gelled substrate as described. ${ }^{6}$ Surface area of the skin substitute was measured over time $(0,1,2,4,6,8,24$, and $48 \mathrm{~h})$ on scale-

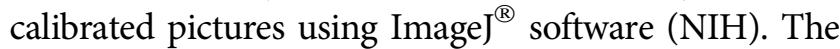
initial area of the die cut $\left(9 \mathrm{~cm}^{2}\right)$ was determined as $100 \%$, and all subsequent measurements were expressed as a ratio to this value. The experiment was repeated twice using newborn or adult cells each time in triplicate.

\section{Grafting on athymic mice}

SASSs were grafted on athymic male nu/nu mice (42 days old) (Charles River Laboratories) as described. ${ }^{14}$ After detaching the skin substitute from its paper frame anchorage, sterile nonadherent gauze was placed on top to facilitate the transfer to the graft bed. Six mice per condition were grafted. Fusenig's silicone chambers were removed after 21 days. For each condition, mice were sacrificed 21 (3 mice) and 90 ( 3 mice) days after grafting. Skin substitute samples (before grafting, 21 and 90 days after grafting) were processed for histological and immunofluorescence analysis. The experiment was repeated three times, each time with SASS-2, SASS3 , and SASS-4 produced with two donor-matched keratinocytes and fibroblasts.

Histological and immunofluorescence analysis Tissue biopsies were fixed overnight in HistoChoice ${ }^{\circledR}$ (Amresco) and embedded in paraffin. Five micrometerthick sections were stained with Masson's trichrome using Weigert's hematoxylin, fuchsin-ponceau, and aniline blue.

For immunodetection, biopsies were embedded in Tissue-Tek OCT Compound (Sakura Finetek) and frozen in liquid nitrogen. Immunofluorescence assays were performed on $5 \mu \mathrm{m}$-thick cryosections fixed with acetone $\left(10 \mathrm{~min}\right.$ at $\left.-20^{\circ} \mathrm{C}\right)$ as previously described. ${ }^{14}$ Cell nuclei were counterstained with Hoechst reagent 33258 (Sigma-Aldrich). The antibodies used were as follows: mouse monoclonal anti-K10 clone RKSE60 (Cedarlane), anti-human filaggrin (Abcam), anti-human K19 clone A53-B/A227 (gift from U. Karsten, Institute of Biological Sciences, University of Rostock, Germany), anti-integrin alpha-3 (VM2) clone HB-8530 (ATCC), anti-laminin-5 (alpha-3 subunit) conjugated to fluorescein isothiocyanate (FITC) (gift from P. Rouselle, IBCP, Lyon), anti-human Ki67 (Pharmingen), anti human leukocyte antigen $A, B$, $\mathrm{C}$ (HLA-ABC), conjugated to FITC (EMD Millipore), and rabbit anti-human type IV collagen (gift from J.A. Grimaud, Pasteur Institute, Lyon, France).

\section{Transmission electron microscopy}

Samples were processed and pictures were acquired as described. ${ }^{15}$ To evaluate the maturation of the basement membrane, a score representing the estimated percentage of the dermoepidermal junction with lamina densa $(1=0-10 \%, 2=11-20 \%, 3=21-30 \%, 4=31-40 \%, 5=$ $41-50 \%, 6=51-60 \%, 7=61-70 \%, 8=71-80 \%, 9=81-$ $90 \%, 10=91-100 \%)$ was given for SASS-2, SASS-3, and SASS-4 specimens from at least four independent experiments.

\section{Results}

The tissue engineering method previously described to produce SASS $-2^{6}$ was compared with two new methods (SASS-3, SASS-4) allowing for a shorter production time. All three types of substitutes have been successfully produced with most keratinocyte and fibroblast populations tested, except with cells from a 30-yearold female donor where only SASS-2 and SASS-4 
have been successfully produced. In this case, the addition of the donor-matched keratinocytes onto the unanchored fibroblast-derived sheet (SASS-3) induced contraction resulting in unusable shrunk tissue.

Macroscopic appearance and histological analysis of SASS cultured in vitro

After 10 days of in vitro maturation at the air-liquid interface, SASS-2, SASS-3 and SASS-4 appeared macroscopically as a tissue that resembled human skin (Fig. 2A-C, respectively). Of note, the surface of SASS3 and SASS-4 was more homogeneous compared to that of SASS-2 (Fig. 2, compare B and C with A). Surface uniformity varied between cell donors. Areas appearing denser or brighter were observed (Fig. 3, compare A with B) in a few cases. Again, these irregularities were more common in SASS-2 compared to SASS-3 and SASS-4 for a given combination of keratinocytes and fibroblasts (Fig. 3, compare B and C with A).
Histological analyses of SASS-2, SASS-3, and SASS-4 revealed the presence of epidermis covering the dermal component at 10 days of culture at the air-liquid interface for all tested conditions. The four typical layers of human epidermis (stratum basale, spinosum, granulosum, and corneum) were observed in denser areas of SASS, indicating a fully differentiated epidermis (Fig. 2D-F, respectively). For less homogeneous SASS, areas that appeared brighter macroscopically were typically associated with a lower number of keratinocyte layers and the absence of a stratum corneum (Fig. 3D), while denser areas were rather associated to a fully differentiated epidermis with squame accumulation (as observed in Fig. 2D-F, arrows).

Some epithelial cell inclusions were seldom observed within the dermal component of SASS (Fig. 3E). Some of these structures were in contact with the epidermis forming invaginations (data not shown). The occurrence of epithelial inclusion was higher within SASS3 and SASS- 4 compared with SASS- 2 .

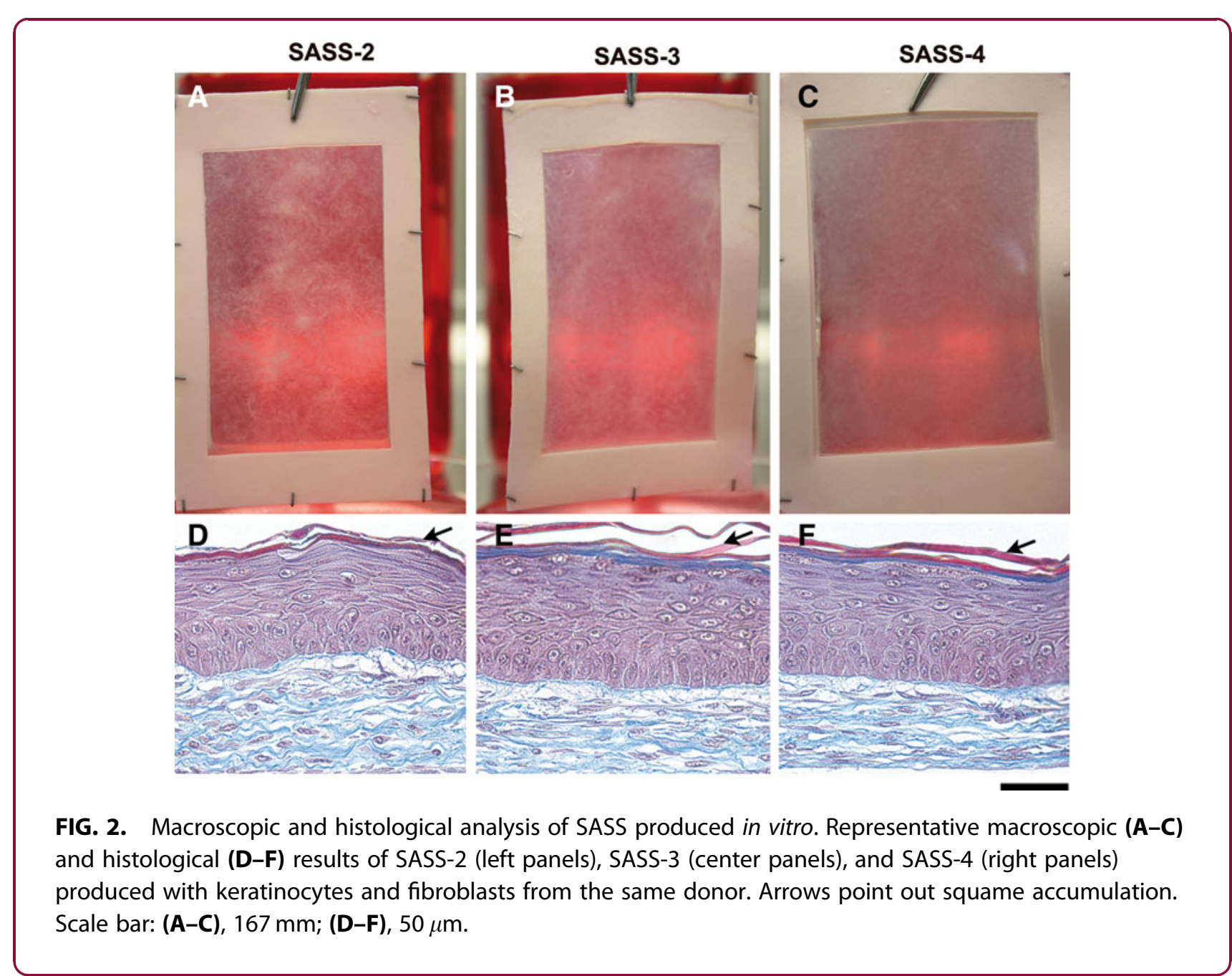




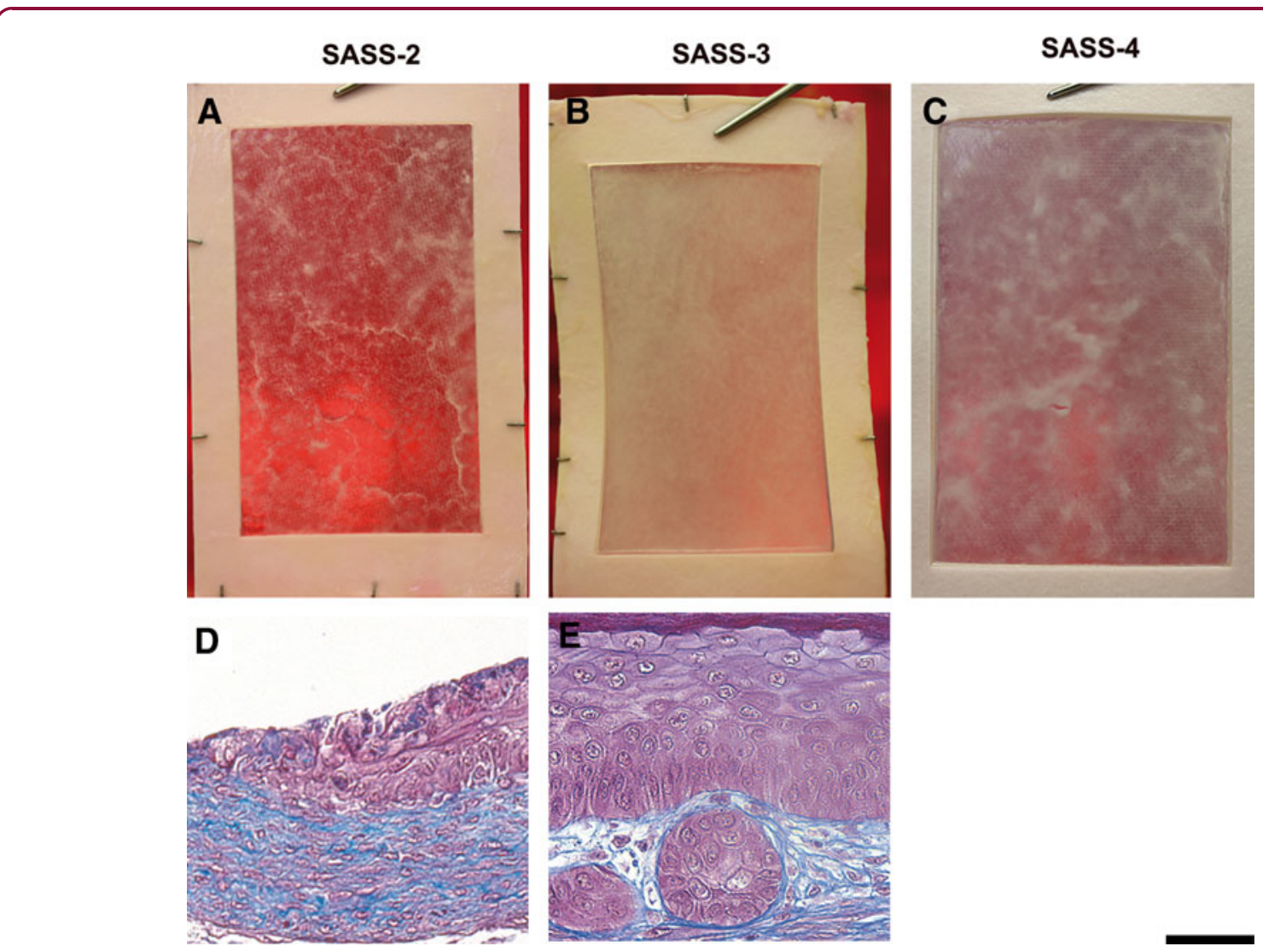

FIG. 3. Macroscopic and histological analysis of SASS produced in vitro. Representative macroscopic (A-C) and histological (D, E) results of SASS-2 (left panels), SASS-3 (center panels), and SASS-4 (right panel) produced with keratinocytes and fibroblasts from the same donor (different from the donor of Fig. 1). Representative histological aspect of an area appearing brighter macroscopically (D) and an epithelial cell inclusion (E). Scale bar: (A-C), $167 \mathrm{~mm}$; (D, E), $50 \mu \mathrm{m}$.

\section{Immunofluorescence analysis}

The proliferation marker Ki-67 was detected in the nucleus of many cells within the stratum basale of SASS2, SASS-3, and SASS-4 (Fig. 4A, B, arrows, and data not shown), indicating that keratinocytes proliferate in the epidermis.

The expression of skin differentiation markers (K19, K14, K10, filaggrin) was similar between SASS-2, SASS3 (Fig. 4), and SASS-4 (data not shown). Keratin (K) 19 immunostaining was performed to evaluate the persistence of stem cells and confirmed the presence of a small subset of basal keratinocytes expressing K19 (Fig. 4C, D, arrows). Basal cells expressed K14 and integrin alpha-3 (data not shown), whereas K10 expression was restricted to the suprabasal layers (Fig. 4E, F, arrows). Filaggrin, a protein associated to the cornified envelope, was detected in the upper cell layers of the epidermis (Fig. 4G, H, arrows). Laminin-5 and type IV collagen, two basement membrane components, were detected as a continuous line at the level of the dermoepidermal junction within SASS-2, SASS-3, and SASS-4 (Fig. 4I-L).

Ultrastructural analysis confirmed the presence of typical structures of the basement membrane such as lamina densa, lamina lucida, and hemidesmosomes for SASS-2, SASS-3, and SASS-4 (Fig. 4M, N and data not shown). The formation of the basement membrane tended to be more advanced in SASS-3 and SASS-4 than in SASS-2 (Table 1).

\section{Tissue contraction of SASS}

The final SASS surface area available for grafting depends on the contraction of the tissue after detachment from the peripheral anchorage at the end of the culture. The structural stability of the skin substitutes after 
Table 1. Maturation of the Basement Membrane Within SASS After 10 Days of Culture at the Air-Liquid Interface
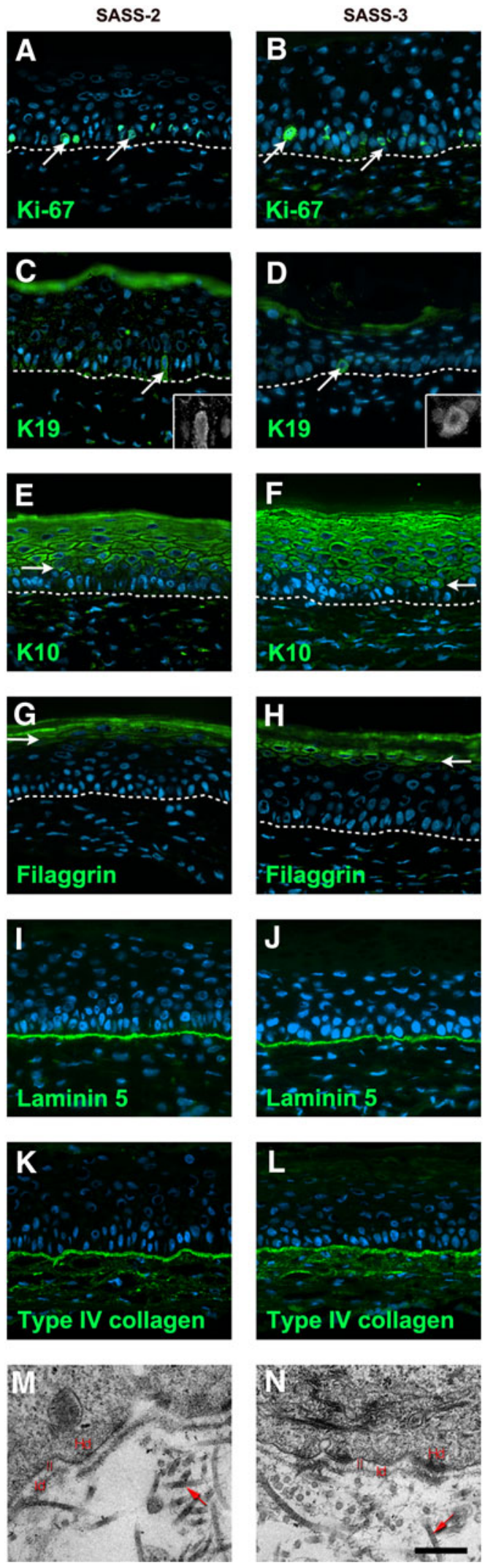

\begin{tabular}{lcccc}
\hline & Mean (SD) & Median & Min & Max \\
\hline SASS-2 $(n=4)$ & $3.8(2.1)$ & 3.5 & 2 & 6 \\
SASS-3 $(n=4)$ & $6.0(3.2)$ & 6.5 & 2 & 9 \\
SASS-4 $(n=4)$ & $6.0(2.7)$ & 7.0 & 2 & 8
\end{tabular}

The score assigned represents the estimated percentage of the dermoepidermal junction with lamina densa $(1=0-10 \%, 2=11-20 \%$, $3=21-30 \%, 4=31-40 \%, 5=41-50 \%, 6=51-60 \%, 7=61-70 \%, 8=71-$ $80 \%, 9=81-90 \%, 10=91-100 \%)$. SASS-3 and SASS-4 were compared to SASS-2 using bilateral Student's $t$-test, and no significant difference was found $(p \geq 0.05)$.

SASS, Self-Assembled Skin Substitute; SD, standard deviation.

detachment from the anchorage was evaluated in vitro over a period of 2 days. Results showed that the contraction kinetic displayed the characteristic exponential decay profile of tissue-engineered skin produce by selfassembly, ${ }^{6}$ independently of the method used for fabrication (Fig. 5). Most of the contraction occurred within $2 \mathrm{~h}$. SASS- 2 tended to contract more than SASS-3 and SASS-4 (Fig. 5).

\section{In vivo maturation of SASS after grafting onto} athymic mice

To validate engraftment capacity and permanent integration, SASS-2, SASS-3 and SASS-4 were transplanted on athymic mice for 90 days. Complete take of all grafts to the wound bed was observed. The epithelium was present on all grafts for the entire duration of the

FIG. 4. Analysis of skin markers and of the ultrastructure of SASS matured in vitro.

Representative pictures of SASS-2 (left panels) and SASS-3 (right panels) produced with keratinocytes and fibroblasts immunolabeled for the detection of Ki-67 (A, B, arrows), K19 (C, D, arrows, inset: high magnification of the cell pointed by the arrow), K10 (E, F, arrows point toward the first labeled cell layer), filaggrin $(\mathbf{G}, \mathbf{H}$, arrows point toward the first labeled cell layer), laminin-5 (I, J), and type IV collagen ( $\mathbf{K}, \mathbf{L})$. Representative transmission electron microscopy observations ( $\mathbf{M}, \mathbf{N}$ ). Cell nuclei were stained in blue with Hoechst reagent (A-L). Dotted line indicates the dermoepidermal junction. Red arrows point to collagen fibers $(\mathbf{M}, \mathbf{N})$. $\mathbf{H d}$, hemidesmosomes; II, lamina lucida; Id, lamina densa. Scale bar: (A-L), $50 \mu \mathrm{m}$; $(\mathbf{M}, \mathbf{N}), 310 \mathrm{~nm}$. 


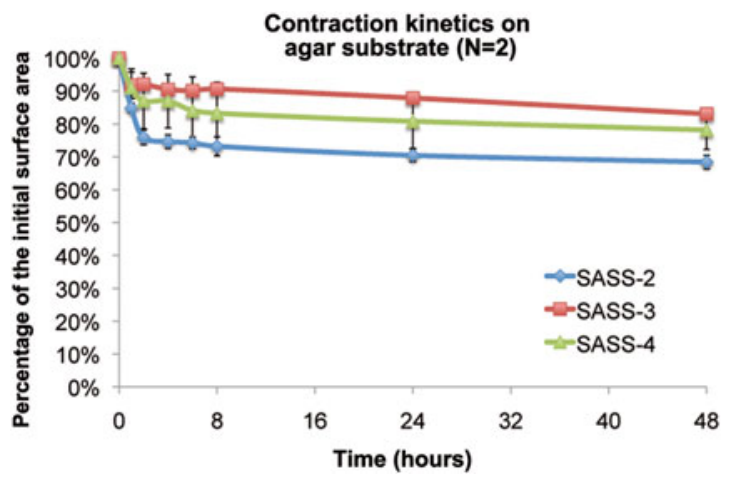

FIG. 5. Contractile behavior of SASS matured in vitro. SASS-2 (blue curve), SASS-3 (red curve), and SASS-4 (green curve) were placed on an agar substrate, and the surface area of the skin substitutes was measured over time to obtain contraction kinetic curves. $\mathrm{N}$ : number of independent experiments performed in triplicate.

experiment, indicating the preservation of functional epithelial stem cells within the skin substitute and longterm epithelial regeneration. During the 21-day period of in vivo maturation in the Fusenig's chamber, SASS2, SASS-3, and SASS-4 surface areas remained stable (data not shown). Three months after grafting, SASS2, SASS-3 and SASS-4 appeared macroscopically as a uniform and smooth opaque tissue much alike human skin (Fig. 6A-C).

Histological analysis revealed that the evolution of SASS-2, SASS-3, and SASS-4 was similar; the epidermis was well organized with all histological layers present 90 days after grafting (Fig. 6D-F). The persistence of human cells after grafting was confirmed by labeling the human histocompatibility complex HLA-ABC (Fig. 6G).

\section{Discussion}

In this study, alternative methods to produce highquality autologous human skin substitutes suitable for the permanent coverage of full-thickness skin wounds were proposed. The three designs derive from the selfassembly approach originally presented by our team for blood vessels, ${ }^{5}$ followed by skin in $1999 .{ }^{9}$ The proposed modifications allow adaptation of the manufacturing process that takes into account the patient variability of intrinsic fibroblast properties such as contractile potential and extracellular matrix secretion. Method SASS-3 should be prioritized because it allows the production of skin substitutes in 31 days, which is 2 weeks less than the previous 45 days required for SASS-2, while SASS- 4 method manages contractile fibroblasts.

Intrinsic contractile properties of fibroblasts as well as their ability to secrete extracellular matrix vary between donors (personal observation). It happens that unanchored fibroblast-derived tissue sheets spontaneously detached from the culture plate after being in culture. Also, the addition of keratinocytes onto an unanchored fibroblast sheet sometimes induces contraction of the sheet followed by their spontaneous detachment and further tissue shrinkage. The SASS-4 method was designed to circumvent this problem by adding a peripheral anchorage to limit spontaneous tissue contraction. Our results are consistent with the limited tissue contraction observed with anchored adipose tissue sheets or myofibroblast-derived tissue sheets. ${ }^{12,16}$

For SASS production to treat a patient, fibroblasts are used from passage 2 . To determine the potential of fibroblasts to generate tissues that resist to manipulation and contraction, a dozen of fibroblast-derived tissue sheets can be prepared from passage 1 fibroblasts to anticipate the behavior of cells, to adjust the optimal culture time, and to orient the choice of the production method to use thereafter.

Epithelial cell inclusions sometimes observed within SASS-2, SASS-3, and SASS-4 were reported to occur in other models of skin substitutes cultured in vitro. ${ }^{17-19}$ In all instances, inclusions disappear following skin graft integration and maturation and are not considered to be clinically significant. ${ }^{17-19}$

The quality of the basement membrane and its adequate ultrastructure in SASS may allow for the stem cell preservation since the basement membrane is involved in the maintenance of epithelial stem cells. ${ }^{20,21}$ In our study, we observed that the formation of the basement membrane was more advanced in SASS-3 and SASS-4 compared with SASS-2. The direct seeding of keratinocytes onto a fibroblast-derived tissue sheet still attached to the bottom of the culture plate ensures an optimal distribution of keratinocytes that probably explains the more homogeneous morphogenesis of the tissue with SASS-3 or SASS-4 methods.

Another explanation of this delayed formation of the basement membrane is based on the mechanosensitivity of human keratinocytes. Previous researchers have reported relations between substrate stiffness and keratinocyte behavior. ${ }^{22-24}$ At the time of keratinocyte adhesion, and linked to the production method, mechanical properties of the interface between cells and the underlying extracellular matrix could be different in SASS-3 and 


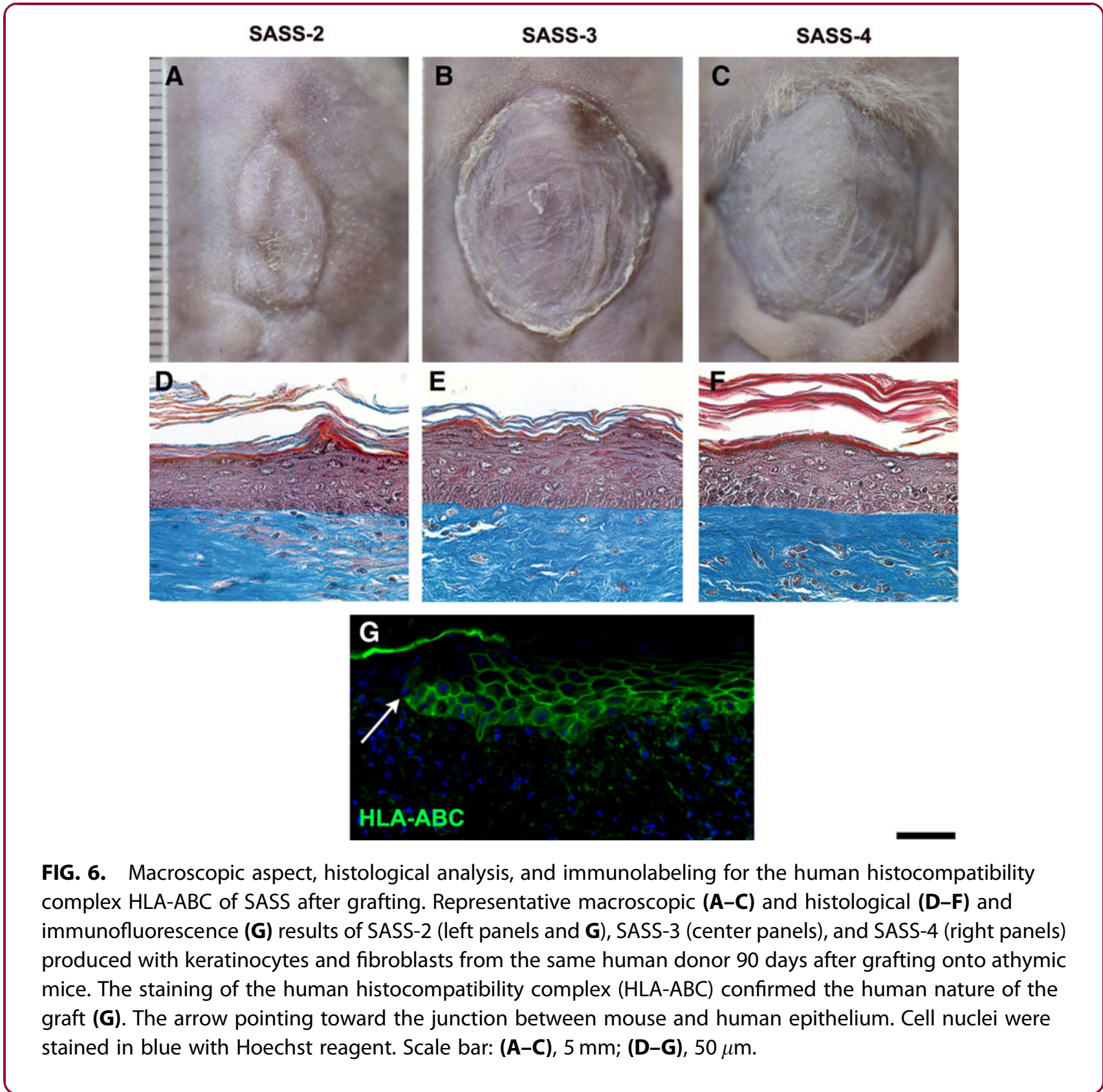

SASS- 4 when compared with SASS-2, and thus, differentially stimulate keratinocytes.

\section{Conclusions}

Our new production methods for SASS allow for the fabrication of high-quality skin substitutes. They are designed for the permanent coverage of full-thickness wounds to replace both the dermis and the epidermis in a single-step grafting procedure. SASS-2, SASS-3, or SASS-4 possesses many desired features such as an autologous epithelium, a stratum corneum, a base- ment membrane at the dermoepidermal junction, a collagen-rich dermal component, mechanical stability, and permanent epithelialization after grafting. A clinical trial assessing the efficacy of SASS for the permanent coverage of full-thickness wounds is currently ongoing in Canada (ClinicalTrials.gov NCTNCT023 50205).

\section{Acknowledgments}

The authors would like to thank Anne-Marie Moisan, Véronique Racine, Cindy Perron, and Sarah-Anne Têtu 
for technical assistance and all LOEX research assistants that contributed to the optimization of the protocols. They are also grateful to Friederike Pfau for the revision of the article. This work was supported by the Fondation des Pompiers du Québec pour les Grands Brûlés (FPQGB), Le Réseau de Thérapie Cellulaire et Tissulaire du FRQS (ThéCell), Canada Research Chair (Tier 1) on Stem Cells and Tissue Engineering (L.G.), Research Chair on Tissue-Engineered Organs and Translational Medicine of the Fondation de l'Université Laval (L.G.), Canadian Institutes of Health Research (CIHR) grant MOP-14364 (F.A.A.), MOP-115093 (F.A.A. and V.J.M.), MOP-12087 (L.G.), and FDN-143213 (L.G.).

\section{Author Contributions}

R.G.1, I.M., R.G.2, D.L., F.A.A., V.J.M., and L.G. contributed substantially to conception, design, and development of the methodology. M.D., R.G.1, I.M., A.A., L.C.-W., A.L., and R.G.2 carried out experiments or have collected data. D.L. wrote the article. D.L., F.A.A., V.J.M., and L.G. supervised the experiments. R.G.1, R.G.2, F.A.A., V.J.M., and L.G. revised the article. F.A.A., V.M., and L.G. were cosenior authors.

\section{Author Disclosure Statement}

No competing financial interests exist.

\section{References}

1. Gomez C, Galan JM, Torrero V, et al. Use of an autologous bioengineered composite skin in extensive burns: clinical and functional outcomes. A multicentric study. Burns. 2011;37:580-589.

2. Llames S, Garcia E, Garcia V, et al. Clinical results of an autologous engineered skin. Cell Tissue Bank. 2006;7:47-53.

3. Boyce ST, Kagan RJ, Greenhalgh DG, et al. Cultured skin substitutes reduce requirements for harvesting of skin autograft for closure of excised, full-thickness burns. J Trauma. 2006;60:821-829.

4. Auger FA, Berthod F, Moulin V, et al. Tissue-engineered skin substitutes from in vitro constructs to in vivo applications. Biotechnol Appl Biochem. 2004;39:263-275.

5. L'Heureux N, Paquet S, Labbe R, et al. A completely biological tissueengineered human blood vessel. FASEB J. 1998;12:47-56.

6. Gauvin R, Larouche D, Marcoux $\mathrm{H}$, et al. Minimal contraction for tissueengineered skin substitutes when matured at the air-liquid interface. J Tissue Eng Regen Med. 2013;7:452-460.

7. Lavoie A, Fugere C, Beauparlant A, et al. Human epithelial stem cells persist within tissue-engineered skin produced by the self-assembly approach. Tissue Eng Part A. 2013;19:1023-1038.

8. Duranceau L, Genest H, Bortoluzzi $P$, et al. Successful grafting of novel autologous tissue-engineered skin substitutes (dermis and epidermis) on twelve burn patients. J Burn Care Res. 2014;35:S121.

9. Michel M, L'Heureux N, Pouliot R, et al. Characterization of a new tissueengineered human skin equivalent with hair. In Vitro Cell Dev Biol Anim. 1999;35:318-326.

10. Larouche D, Paquet C, Fradette J, et al. Regeneration of skin and cornea by tissue engineering. Methods Mol Biol. 2009;482:233-256.

11. Bisson $F$, Rochefort $E$, Lavoie $A$, et al. Irradiated human dermal fibroblasts are as efficient as mouse fibroblasts as a feeder layer to improve human epidermal cell culture lifespan. Int J Mol Sci. 2013;14:4684-4704.

12. Corriveau MP, Boufaied I, Lessard J, et al. The fibrotic phenotype of systemic sclerosis fibroblasts varies with disease duration and severity of skin involvement: reconstitution of skin fibrosis development using a tissue engineering approach. J Pathol. 2009;217:534-542.

13. Trottier V, Marceau-Fortier G, Germain L, et al. IFATS collection: using human adipose-derived stem/stromal cells for the production of new skin substitutes. Stem Cells. 2008;26:2713-2723.

14. Pouliot R, Larouche D, Auger FA, et al. Reconstructed human skin produced in vitro and grafted on athymic mice. Transplantation. 2002;73:1751-1757.

15. Simard-Bisson C, Bidoggia J, Larouche D, et al. A role for DLK in microtubule reorganization to the cell periphery and in the maintenance of desmosomal and tight junction integrity. J Invest Dermatol. 2016 [Epub ahead of print]; DOI: 10.1016/j.jid.2016.07.035.

16. Vermette $M$, Trottier V, Menard V, et al. Production of a new tissueengineered adipose substitute from human adipose-derived stromal cells. Biomaterials. 2007;28:2850-2860.

17. Beaudoin Cloutier C, Guignard R, Bernard G, et al. Production of a bilayered self-assembled skin substitute using a tissue-engineered acellular dermal matrix. Tissue Eng Part C Methods. 2015;21:1297-1305.

18. Butler CE, Orgill DP, Yannas IV, et al. Effect of keratinocyte seeding of collagen-glycosaminoglycan membranes on the regeneration of skin in a porcine model. Plast Reconstr Surg. 1998;101:1572-1579.

19. Compton CC, Gill JM, Bradford DA, et al. Skin regenerated from cultured epithelial autografts on full-thickness burn wounds from 6 days to 5 years after grafting. A light, electron microscopic and immunohistochemical study. Lab Invest. 1989;60:600-612.

20. Jones PH, Watt FM. Separation of human epidermal stem cells from transit amplifying cells on the basis of differences in integrin function and expression. Cell. 1993;73:713-724.

21. Brizzi MF, Tarone G, Defilippi P. Extracellular matrix, integrins, and growth factors as tailors of the stem cell niche. Curr Opin Cell Biol. 2012;24:645-651.

22. Trappmann B, Gautrot JE, Connelly JT, et al. Extracellular-matrix tethering regulates stem-cell fate. Nat Mater. 2012;11:642-649.

23. Zarkoob H, Bodduluri S, Ponnaluri SV, et al. Substrate stiffness affects human keratinocyte colony formation. Cell Mol Bioeng. 2015;8:32-50.

24. Gupta A, Jain G, Kaur M, et al. Association of impaired baroreflex sensitivity and increased arterial stiffness in peritoneal dialysis patients. Clin Exp Nephrol. 2016;20:302-308.

Cite this article as: Larouche $D$, Cantin-Warren $L$, Desgagné $M$, Guignard R, Martel I, Ayoub A, Lavoie A, Gauvin R, Auger FA, Moulin VJ, Germain L (2016) Improved methods to produce tissueengineered skin substitutes suitable for the permanent closure of fullthickness skin injuries, BioResearch Open Access 5:1, 320-329, DOI: 10.1089/biores.2016.0036
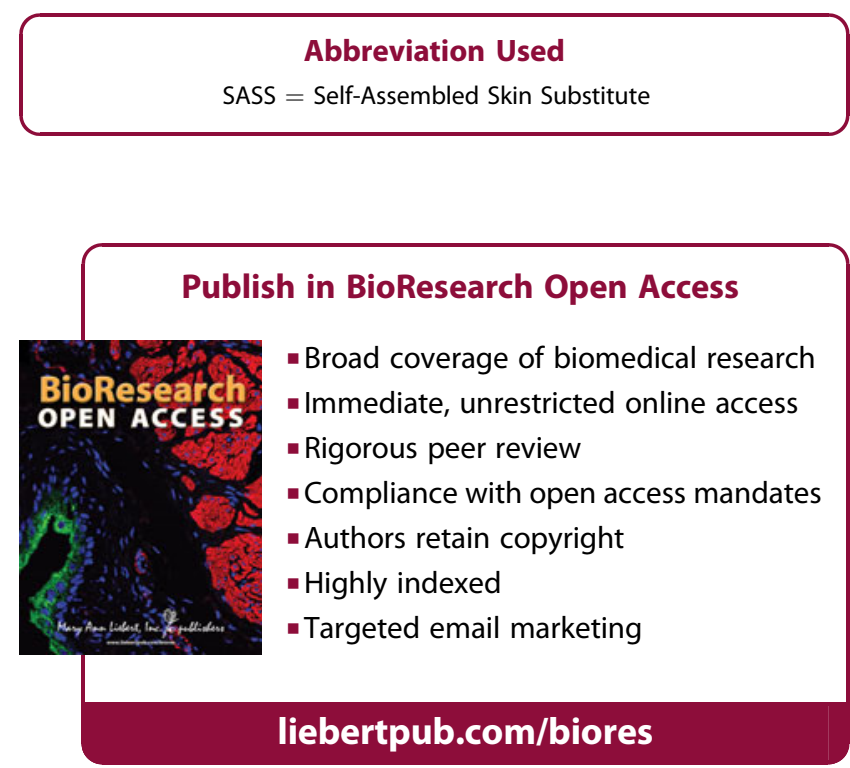